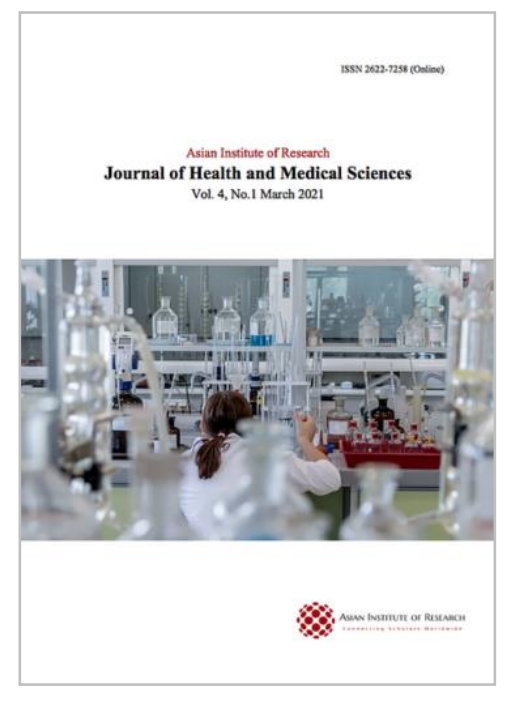

\title{
Journal of Health and Medical Sciences
}

\begin{abstract}
Almawazini Abdulmajid, Alnashi Sinan, Alsharkawy Ali, Taha Sami Ahmed, Alghamdi Abdullah Ismail Saeed, Alghamdi Omair Mohammed Saad, Alzahrani Ahmed Ibrahim Ahmed, Alghamdi Abdulmalik Ahmed Abdullah, Almawazini Hazem, Almawazini Mohammad, and Shumrani Khalid Mohammad. (2021), Review of Arrhythmias in Children with Congenital Heart Disease in Albaha Area, Saudi Arabia. In: Journal of Health and Medical Sciences, Vol.4, No.1, 1822.
\end{abstract}

ISSN 2622-7258

DOI: 10.31014/aior.1994.04.01.151

The online version of this article can be found at: https://www.asianinstituteofresearch.org/

Published by:
The Asian Institute of Research

The Journal of Health and Medical Sciences is an Open Access publication. It may be read, copied, and distributed free of charge according to the conditions of the Creative Commons Attribution 4.0 International license.

The Asian Institute of Research Journal of Health and Medical Sciences is a peer-reviewed International Journal. The journal covers scholarly articles in the fields of Medicine and Public Health, including medicine, surgery, ophthalmology, gynecology and obstetrics, psychiatry, anesthesia, pediatrics, orthopedics, microbiology, pathology and laboratory medicine, medical education, research methodology, forensic medicine, medical ethics, community medicine, public health, community health, behavioral health, health policy, health service, health education, health economics, medical ethics, health protection, environmental health, and equity in health. As the journal is Open Access, it ensures high visibility and the increase of citations for all research articles published. The Journal of Health and Medical Sciences aims to facilitate scholarly work on recent theoretical and practical aspects of Health and Medical Sciences. 
The Asian Institute of Research Journal of Health and Medical Sciences

Vol.4, No.1, 2021: 18-22 ISSN 2622-7258

\title{
Review of Arrhythmias in Children with Congenital Heart Disease in Albaha Area, Saudi Arabia
}

\author{
Abdulmajid Almawazini ${ }^{1}$, Sinan Alnashi ${ }^{1}$, Ali Alsharkawy ${ }^{1}$, Sami Ahmed Taha ${ }^{1}$, Abdullah Ismail Saeed \\ Alghamdi ${ }^{2}$, Omair Mohammed Saad Alghamdi ${ }^{2}$, Ahmed Ibrahim Ahmed Alzahrani², Abdulmalik Ahmed \\ Abdullah Alghamdi ${ }^{2}$, Hazem Almawazini ${ }^{3}$, Mohammad Almawazini ${ }^{4}$, Khalid Mohammad Shumrani ${ }^{5}$
}

\author{
${ }^{1}$ Consultant, pediatric department, King Fahad Hospital Albaha, KSA \\ ${ }^{2}$ Pediatric resident, King Fahad Hospital Albaha, KSA \\ ${ }^{3}$ Medical student, Cairo University Egypt \\ ${ }^{4}$ Master degree, Bradford University, UK \\ ${ }^{5}$ Faculty of Medicine, Albaha University
}

Correspondence: Abdulmajid Almawazini, PhD. MD. Consultant pediatric cardiologist, KFH Albaha. Mail address: Building 7223. unit 2. Albaha. Zip code 65732. Additional No 4719. amawazini@gmail.com. Mobile: 00966508294471. http://orcid.org/0000-0002-9303-6015

\begin{abstract}
Background: Arrhythmias are common in children with congenital heart disease (CHD) and contribute to morbidity and mortality. The prevalence, type, and presentation vary depending age and other factors. Objectives: This study aimed to determine the prevalence of arrhythmias and associated factors in children with CHD in King Fahad Hospital, Al Baha, Saudi Arabia. Methods: This was a hospital-based retrospective crosssectional study conducted from January 2010 to December 2020. In total, 185 patients, aged 1 day to 14 years, were included. Children with arrhythmias and CHD were included, while those without CHD were excluded. Data were analyzed using SPSS version 16.0. Results: Arrhythmias were confirmed in 46 patients $(24.86 \%)$, while 139 patients $(75.14 \%)$ had no electrocardiographic abnormalities, $95 \%$ CI (14.12-21.22). Among the arrhythmias, we found that first-degree atrio-ventricular block was the most common and reported in 30 patients $(16.21 \%)$; atrial tachyarrhythmias in 5 patients $(2.7 \%)$; premature ventricular contractions in 4 patients $(2.16 \%)$; premature atrial contractions in 3 patients $(1.62 \%)$; sinus bradycardia in 2 patients $(1.1 \%)$; complete heart block in 1 patient and a prolonged QT interval with severe hypokalemia, $((0.54 \%)$ for each. Conclusion: Arrhythmias are common in children with CHD, and early diagnosis and regular follow-up have significant benefits in management. Cardiac surgery, heart failure, digoxin, and electrolyte disturbances were confirmed as risk factors. Article
\end{abstract}

Keywords: Children, Congenital Heart Disease, Arrhythmia

\section{Abbreviations}

CHD: congenital heart disease; VSD: ventricular septal defect; ASD: atrial septal defect; AV: atrio-ventricular; AVC: atrio-ventricular canal; ECG: electrocardiography; TGA: transposition of great arteries; DORV: double outlet right ventricle; PACs: premature atrial contractions; PVCs: premature ventricular contractions; TOF: Tetralogy of Fallot; CI: confidence interval; KFH: King Fahad Hospital. 


\section{Background}

Children with congenital heart disease (CHD) develop many complications such as failure to thrive, heart failure, cyanosis, and arrhythmias [Ronald Wells M.D. Paul Khairy MD etal.]. Arrhythmias are associated with severe cardiac dysfunction. Structural abnormalities and conductive tissue dysfunction are important factors that predispose children with CHD to cardiac arrhythmias, which can affect their morbidity and mortality. The clinical presentation of arrhythmias can differ depending on age, metabolism of cardiac tissue, hemodynamic changes, and the type of CHD [Reena M Ghosh, Gregory J Gates etal, Antonio Herna'ndez-Madrid e tal]. The risk of atrial fibrillation can be increased with long PR intervals and other types of atrial dysrhythmias [Arsha Karbassi, Krishnakumar Nair etal, Cheng S, Keyes MJ etal]. Atrial tachyarrhythmia is more commonly associated with adult CHD and is one of the most common complications [Cheng S, Keyes MJ etal]. Many normal healthy children develop supraventricular tachycardia, and physicians should be familiar with its presentation and management [Schlechte EA, Boramanand $\mathrm{N}$ etal]. Electrolyte imbalances and poor nutritional status can also increase the risk of arrhythmias [World Health Organization]. In addition, hemodynamic changes increase the risk of atrial and ventricular arrhythmias associated with CHD. A wide range of arrhythmias can be present in CHD, and each one needs to be well managed. Ablation can be performed in unresponsive cases of arrhythmias [Charlotte A Houck, Stephanie F Chandler etal]. Arrhythmias are also frequent in children who have undergone cardiac surgery. Age $>5$ years, low weight, long bypass time, electrolyte imbalances, use of digoxin, and use of inotropes are considered risk factors for postoperative arrhythmias [Anthony Batte, Peter Lwabi etal, Tabitha G Moe, Victor A Abrich etal]. In addition, the type of surgical procedure, irritation of the conductive tissue by humoral factors during surgery, postoperative scarring, high serum lactate levels, and hypotension during and after surgery increase the incidence of arrhythmias [Tarek Ahmed Abdel Gawad etal, Lars Grosse-Wortmann, Suzanna Kreitz etal, Sahu MK, Das A etal, Grosse-Wortmann L, Kreitz S etal]. Early diagnosis of CHD are important in the management of associated arrhythmias [Mocumbi AO, Lameira E etal].

\section{Objectives and methods}

This study aimed to determine the prevalence of and risk factors associated with arrhythmias among children with CHD. The study was approved by the Ethical Research Committee in King Fahad Hospital (KFH), Al Baha, Saudi Arabia. This was a retrospective hospital-based cross-sectional study conducted in the pediatric and neonatology departments of KFH from January 2010 to December 2020. The sample size needed was calculated using the Leslie Kish formula [Jon Wiley and Sons INC]. Total of 185 cases were reviewed for the presence of CHD and arrhythmias. The patients' ages ranged between 1 day and 14 years. Patients with CHD who had arrhythmias were included, and children with normal heart structures were excluded. Clinical examination, electrocardiography (ECG), and echocardiography were performed by a pediatric cardiologist. Holter ECG was performed in some cases to confirm the diagnosis. A complete blood count was performed and serum electrolyte levels were assessed for each child. The type of CHD, the type of arrhythmia, surgical procedures performed, patient age, sex, electrolyte imbalances, and use of certain medications were all evaluated. A thyroid function test performed in cases of tachy and bradyarrhythmias. Statistical analyses were performed using SPSS version 16.0.

\section{Results}

As shown in Table1-2, we reviewed 185 medical records of children diagnosed with CHD, aged 1 day to 14 years. The majority of the children, $105(56.76 \%)$, were female, and 80 (43.24\%) were male. Ventricular septal defect (VSD) was the most common type of CHD, seen in 55 of the cases (29.73\%), followed atrial septal defect in $37(20 \%)$, patent ductus arteriosus in 35 (18.92\%), tetralogy of Fallot (TOF) in 20 (10.81\%), atrio-ventricular canal (AVC) in $11(5.95 \%)$, mitral valve prolapse in $10(5.41 \%)$, pulmonary stenosis in $7(3.78 \%)$, aortic stenosis in $4(2.16 \%)$, d-transposition of great arteries (TGA) in $3(1.62 \%)$, double outlet right ventricle in 2 $(1.1 \%)$, and Ebstein anomaly in $1(0.54 \%)$. Arrhythmias were found in 46 patients $(24.86 \%)$, while no arrhythmias were found in 139 patients $(75.14 \%)$, $95 \%$ confidence interval (CI) (14.12-21.22). First-degree atrio-ventricular heart blocks were diagnosed in 30 patients (16.21\%), $95 \%$ CI (12.8-21.6). It was more common in patients older than five years and was increased with the use of medication such as digoxin, and the 
presence of hypokalemia (OR 3.75), $95 \%$ CI (11.60-18.86). Atrial tachyarrhythmias in 5 patients (2.7\%), $95 \%$ CI (11.2-16.22), premature ventricular contractions (PVCs) in 4 patients (2.16\%), $95 \%$ CI (10.2-17.12), premature atrial contractions (PACs) in 3 patients (1.62\%), 95\% CI (9.5-17.5), sinus bradycardia in 2 patients (1.1\%) 95\% CI (8.15-18.5), complete heart block in 1 patient (0.54\%), $95 \%$ CI (2.7-6.6), and prolonged QT interval associated with severe hypokalemia in 1 patient $(0.54 \%)$. Electrolyte abnormalities were confirmed in few cases, and it was associated with in premature extrasystole contraction $(P=0.008)$.

\section{Discussion}

Multiple structural heart anomalies were observed in the patients. In this study, arrhythmias were found in $46 / 185$ patients $(24.86 \%)$ of children with congenital heart disease, while in $139 / 185$ (75.14\%) patients, no arrhythmias were documented, as mentioned in many published articles [Tabitha G MoeTarek Ahmed etal, Abdel Gawad etal, Lars Grosse-Wortmann, Suzanna Kreitz etal]. The high prevalence of first-degree heart blocks in this study was compatible with the results of other international studies [Arsha Karbassi, Krishnakumar Nair etal, Cheng S, Keyes MJ etal, Grosse-Wortmann L, Kreitz S etal, Mocumbi AO, Lameira E etal]. The majority of patients underwent palliative corrective heart surgery, and no electrolyte abnormalities were observed. As mentioned in the literature, first-degree AV block was considered a benign condition in children, but it has also been associated with an increased risk of atrial fibrillation [Cheng S, Keyes MJ etal, Tabitha G Moe, Victor A Abrich etal]. Atrial tachyarrhythmia was confirmed in 5 patients (2.7\%). In 4 patients $(2.16 \%)$ who had VSD, PVCs were diagnosed. The prevalence of PVCs was reported to be $1.5 \%$ among children with CHD in other published studies [World Health Organization paper]. In 3 patients (1.62\%) with PACs reported, 2 had TOF, and the third had a complete AVC. Additionally, in those 3 patients, laboratory results were normal in 2, while the third patient had hypokalemia [Tabitha G Moe, Victor A Abrich etak\#l, Anthony Batte, Peter Lwabi etal]. Two patients (1.1\%) had sinus bradycardia. There are limited data available on this issue. Complete heart block was confirmed in 1 patient $(0.54 \%)$ who underwent TOF repair with normal laboratory workup, which was comparable to other international studies. A prolonged QT interval with severe hypokalemia in reported with 1 patient $(0.54 \%)$. Holter ECG was helpful for diagnosis in two cases [Anthony Batte, Peter Lwabi etal, Sahu MK, Das A, Siddharth B etal]. These results indicate that ECG should be performed regularly in children with CHD. In this study, we found that children aged 6 years and older were more likely to have firstdegree heart block associated with digoxin, compared to children younger than this age, who can tolerate more before the manifestations of digoxin toxicity occur.

\section{Study limitations}

No genetic investigations were performed to evaluate the genetic contribution of arrhythmias associated with CHD.

\section{Conclusion}

Arrhythmias are relatively common among children with CHD in the Al Baha area of Saudi Arabia. This study found that the type of CHD and surgical procedure, age, digoxin use, and electrolyte imbalances were important factors that need to be assessed among children with CHD. Early diagnosis and management are important.

Table 1: distribution of CHD presented in Children included in the study.

\begin{tabular}{|l|l|l|l|}
\hline Abnormality & Frequency & Percentage & P value \\
\hline Total patients & 185 & $100 \%$ & \\
\hline Ventricular Septal Defect & 55 & $29.73 \%$ & 0.07 \\
\hline Atrial septal defect & 37 & $20 \%$ & 0.08 \\
\hline Patent Ductus Arteriosus & 35 & $18.92 \%$ & 0.08 \\
\hline Tetralogy of Fallot & 20 & $10.81 \%$ & 0.09 \\
\hline Complete atrio-ventricular defect & 11 & $5.95 \%$ & 0.06 \\
\hline Mitral valve prolapse & 10 & $5.41 \%$ & 0.07 \\
\hline Pulmonary stenosis & 7 & $3.78 \%$ & 0.05 \\
\hline Aortic stenosis & 4 & $2.16 \%$ & 0.06 \\
\hline Transposition of Great Arteries & 3 & $1.62 \%$ & 0.05 \\
\hline
\end{tabular}




\begin{tabular}{|l|l|l|l|}
\hline Double outlet right ventricle & 2 & $1.1 \%$ & 0.05 \\
\hline Ebstein anomaly & 1 & $0.54 \%$ & 0.08 \\
\hline
\end{tabular}

Table 2: distribution of arrhythmias found in this study.

\begin{tabular}{|l|l|l|l|}
\hline Arrhythmia & Frequency & Percentage & $(95 \% \mathrm{CI})$ \\
\hline First degree A-V block & 30 & $16.21 \%$ & $(12.8-21.6)$ \\
\hline Atrial tachyarrhythmias & 5 & $2.7 \%$ & $(11.2-16.22)$ \\
\hline Premature ventricular contractions & 4 & $2.16 \%$ & $(10.2-17.12)$ \\
\hline Premature atrial contractions & 3 & $1.62 \%$ & $(9.5-17.5)$ \\
\hline Sinus bradycardia & 2 & $1.1 \%$ & $(8.15-18.5)$ \\
\hline Complete heart block & 1 & $0.54 \%$ & $(11.5-19.5)$ \\
\hline Long QT & 1 & $0.54 \%$ & $(11.5-19.5)$ \\
\hline No arrhythmias confirmed & 139 & $75.13 \%$ & $(14.12-21.22)$ \\
\hline
\end{tabular}

\section{References}

Ronald Wells M.D. Paul Khairy MD. Christian Anderson MD. Louise Hassis M.D. Seshadri Balaji M.B.B.S., M.R.C.P. (U.K.), Ph.D. Dofetilide for Atrial Arrhythmias in Congenital Heart Disease: A Multicenter Study. First published: 25 September 2009. https://doi.org/10.1111/j.1540-8159.2009.02479.

Reena M Ghosh, Gregory J Gates, Christine A Walsh, Myles S Schiller, Robert H Pass, Scott R Ceresnak, DOI: 10.1007/s00246-014-1053-9 The prevalence of arrhythmias, predictors for arrhythmias, and safety of exercise stress testing in children, Pediatr Cardiol. 2015 Mar;36(3):584-90. doi: 10.1007/s00246-014-10539. Epub 2014 Nov 11.

Antonio Herna'ndez-Madrid, Thomas Pau, Dominic Abrams, Peter F. Aziz4, Nico A. Blom, Jian Chen, Massimo Chessa, Nicolas Combes, Nikolaos Dagres, Gerhard Diller, Sabine Ernst, Alessandro Giamberti, Joachim Hebe, Jan Janousek, Thomas Kriebel, Jose Moltedo, Javier Moreno, Rafael Peinado,Laur ent Pison, Eric Rosenthal, Jonathan R. Skinner, and Katja Zeppenfeld. Arrhythmias in congenital heart disease: a position paper of the European Heart Rhythm Association (EHRA), Association for European Paediatric and Congenital Cardiology (AEPC), and the European Society of cardiology (ESC) Working Group on Grown-up Congenital heart disease, endorsed by HRS, PACES, APHRS, and SOLAECE. Europace (2018) 0, 1-35. doi:10.1093/europace/eux380. Published on behalf of the European Society of Cardiology, on 21 March 2018.

Arsha Karbassi, Krishnakumar Nair, Louise Harris, Rachel M Wald, S Lucy Roche. DOI: 10.4330/wjc.v9.i6.496, Atrial tachyarrhythmia in adult congenital heart disease. World J Cardiol. 2017 Jun 26;9(6):496-507. doi: 10.4330/wjc.v9.i6.496.

Cheng S, Keyes MJ, Larson MG, McCabe EL, Newton-Cheh C, Levy D, Benjamin EJ, Vasan RS, Wang TJ. Long-term outcomes in individuals with prolonged PR interval or first-degree atrioventricular block. Jama. 2009;301(24):2571-7.

Schlechte EA, Boramanand N, Funk M. Supraventricular tachycardia in the pediatric primary care setting: Agerelated presentation, diagnosis, and management. J Pediatr Health Care. 2008;22(5):289-99.

World Health Organization: Haemoglobin concentrations for the diagnosis of anaemia and assessment of severity. Vitamin and Mineral Nutrition Information System. Geneva 2011.

Charlotte A Houck, Stephanie F Chandler, Ad J J C Bogers, John K Triedman, Edward P Walsh, Natasja M S de Groot 1, Dominic J Abrams. Arrhythmia Mechanisms and Outcomes of Ablation in Pediatric Patients with Congenital Heart Disease. Circ Arrhythm Electrophysio. https://pubmed.ncbi.nlm.nih.gov/31722541/. Epub 2019 Nov;12(11): e007663. doi: 10.1161/CIRCEP. 119.007663.

Anthony Batte, Peter Lwabi, Sulaiman Lubega, Sarah Kiguli, Violette Nabatte, Charles Karamagi. Prevalence of arrhythmias among children below 15 years of age with congenital heart diseases attending Mulago National Referral Hospital, Uganda. BMC Cardiovasc Disord. 2016 Apr 13; 16:67. doi: 10.1186/s12872016-0243-1.

Tabitha G Moe, Victor A Abrich, Edward K Rhee. Atrial Fibrillation in Patients with Congenital Heart Disease. J Atr Fibrillation Pubmed ecollection, 2017 Jun 30; 10(1):1612. Doi: 10.4022/jafib, 1612. e collection JunJul 2017.

Tarek Ahmed Abdel Gawad, Waleed Mohamed Elguindy, Omneya Ibrahim Youssef, Tamer Ashraf Abosalem. The Prevalence and Risk Factors of Early Arrhythmias Following Pediatric Open Heart Surgery in Egyptian Children, Open Access Maced J Med Sci.2017 Dec 5;5(7):940-944. doi: 10.3889/oamjms.2017.177. eCollection 2017 Dec 15.

Lars Grosse-Wortmann, Suzanna Kreitz, Ralph G Grabitz, Jaime F Vazquez-Jimenez, Bruno J Messmer, Goetz von Bernuth, Marie-Christine Seghaye. Prevalence of and risk factors for perioperative arrhythmias in 
neonates and children after cardiopulmonary bypass: continuous holter monitoring before and for three days after surgery. J Cardiothorac Surg. 2010 Oct 18;5:85. doi: 10.1186/1749-8090-5-85.

Sahu MK, Das A, Siddharth B, Talwar S, Singh SP, Abraham A, Choudhury A.Arrhythmias in Children in Early Postoperative Period After Cardiac Surgery. World J Pediatr Congenit Heart Surg. 2018 Jan;9(1):38-46. doi: $10.1177 / 2150135117737687$.

Grosse-Wortmann L, Kreitz S, Grabitz RG, Vazquez-Jimenez JF, Messmer BJ, von Bernuth G, Seghaye M-C. Prevalence of and risk factors for perioperative arrhythmias in neonates and children after cardiopulmonary bypass: continuous holter monitoring before and for three days after surgery. J Cardiothoracic Surg. 2010; 5(1):85.

Mocumbi AO, Lameira E, Yaksh A, Paul L, Ferreira MB, Sidi D. Challenges on the management of congenital heart disease in developing countries. Int J Cardiol. 2011; 148(3):285-8.

Jon Wiley and Sons INC publication; Leslie Kish, University of Michigan; Wiley classics library edition published 2004; Statistical design for research; $\mathrm{N}=\mathrm{t}^{2} \mathrm{PQ} / \mathrm{D}^{2}$. 